\title{
BALKING AND RENEGING OF BATCHES IN VOD APPLICATIONS
}

R. Vanalakshmi

Research Scholar, Department of Mathematics, Kalasalingam Academy of Research and Education.

Krishnankovil, (India).

E-mail: vanalakshmi31@gmail.com ORCID: https://orcid.org/0000-0002-3440-5044

S. Maragathasundari

Associate Professor, Department of Mathematics, Kalasalingam Academy of Research and Education.

Krishnankovil, (India).

E-mail: maragatham01@gmail.com ORCID: https://orcid.org/0000-0003-1210-6411

K. S. Dhanalakshmi

Assistant Professor, Department of Electronics and Communication Engineering,

Kalasalingam Academy of Research and Education.

Krishnankovil, (India).

E-mail: k.s.dhanalakshmi@klu.ac.in ORCID: https://orcid.org/0000-0001-6285-3656

Recepción: 05/12/2019 Aceptación: 08/01/2020 Publicación: 23/03/2020

\section{Citación sugerida:}

Vanalakshmi, R., Maragathasundari, S., y Dhanalakshmi, K. S. (2020). Balking and reneging of batches in vod applications. 3C Tecnología. Glosas de innovación aplicadas a la pyme. Edición Especial, Marzo 2020, 69-89. http://doi.org/10.17993/3ctecno.2020.specialissue4.69-89

\section{Suggested citation:}

Vanalakshmi, R., Maragathasundari, S., \& Dhanalakshmi, K. S. (2020). Balking and reneging of batches in vod applications. 3C Tecnología. Glosas de innovación aplicadas a la pyme. Edición Especial, Marzo 2020, 69-89. http://doi.org/10.17993/3ctecno.2020.specialissue4.69-89 


\section{ABSTRACT}

This paper researches an examination on Video-on-request (VOD) alludes to video benefits in which customers can demand any video program from a server whenever. VOD has significant applications in stimulation, training, data, and promoting, for example, film onrequest, remove learning, home shopping, and intelligent news, so on. Versatility in gushing limit can be cultivated by strategies for interest clumping, in which requests for a video touching base inside a time allotment are collected together (i.e., batched) and presented with a lone multicast stream. The target here is to achieve the trade between the multicasting cost and customer delay in the system. We analyze different clustering schemes (as far as customers concede experienced, the amount of customers assembled in each bunch, thus on...), and how framework benefit can be helped given client's reneging conduct. This issue of postponement in patch up procedure and separate happening in VOD writing computer programs is drawn nearer through lining hypothesis in this investigation. Lining disposition characterizes the mistake I the systems administration and gives out the expected plans to be passed out to limit the blunder happening assets. It likewise presents the idea of support period after the fruition of administration. Numerical delineation and an expand graphical investigation are completed toward the conclusion to approve the model. It gives a reasonable pondered the applied investigation of lining hypothesis in VOD systems.

\section{KEYWORDS}

Balking, Reneging, Batch arrival, Emergency vacation, Compulsory vacation. 


\section{INTRODUCTION}

VIDEO-ON-DEMAND (VOD) spilling administration over remote systems is exponentially expanding with creative advances in keen cell phones. To give the VOD spilling administration, high caliber of administration (QoS)requirements ought to be met, for example, high transfer speed necessity, low administration idleness, low administration blocking rate, and so forth. Also, clients need to access to any substance, whenever, anyplace, on any gadget. The conventional unicast transmission has focal points such straightforwardness, no administration inactivity (i.e., start-up postponement), and simple execution of client heterogeneities, for example, cushion limit and channel conditions. Be that as it may, unicast isn't versatile. The transfer speed utilization increments directly as the client solicitations increment. In this light, a few multicast/communicate based transmission plans are being concentrated to deal with the development of portable video traffic. They are versatile and productive as far as data transmission necessity. All things considered, the administration by and large produces extreme idleness and can't promptly think about client heterogeneities. Clumping, fixing, and occasional telecom plans are in the classes of the multicast/communicate based transmission.

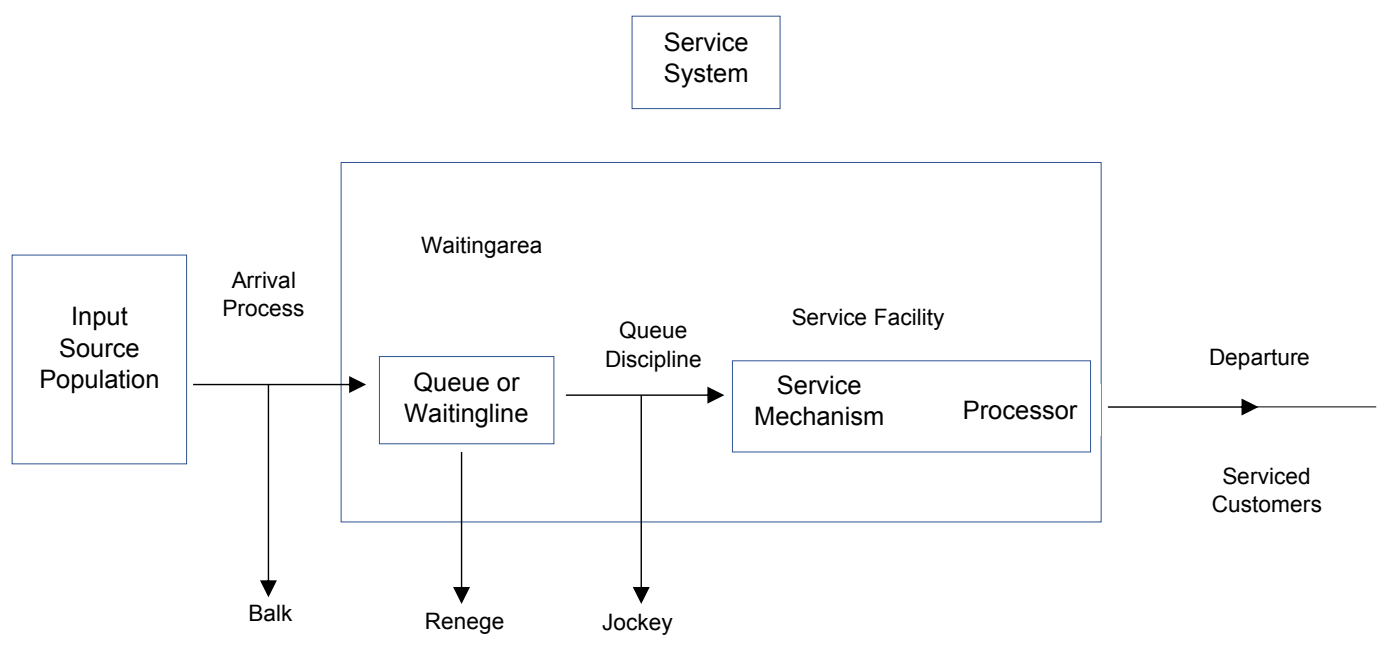

Among them, clumping can ensure the administration inactivity inside specific limits for both mainstream and disliked substance The record related qualities of a VOD application incorporate gushing data transfer capacity, size of the documents, number of video titles, and video fame. The spilling transfer speed of a video, b0, relies upon the video pressure 
plan utilized (e.g., MPEG-I, MPEG-II, movement JPEG, and so forth). It can go from under $1 \mathrm{Mbps}$ to more than $10 \mathrm{Mbps}$. Gushing data transfer capacity likewise relies upon the encoding technique utilized (e.g., Constant-piece rate, Variable-piece rate, etc.). The size of a video document is the genuine extra room the record expends in a capacity medium. It might run from $\sim 10 \mathrm{MB}$ (promoting cuts) to more than $\sim 1$ GB (motion pictures). All documents in a VOD application may not be of a similar size. In MOD (film on-request), for instance, the record size is probably going to be comparable or "homogeneous," with each document of about, state, an hour and a half playback time. Then again, record estimate in intelligent news condition can be fairly "heterogeneous," contingent upon the bit of news and whether it is a narrative or not. Somewhere close to the "limits" might be home shopping, in which record size may run from $\sim 5 \mathrm{MB}$ to $\sim 30 \mathrm{MB}$ (20 seconds to 2 minutes). applications focused to overall population are probably going to have a greater number of titles than applications for a littler gathering of clients. Various recordings have diverse access recurrence. The ubiquity of a video is characterized as the likelihood for the video to be gotten to or picked by any approaching solicitation.

Singh (2016) showed that the gushing design and security issues were the difficulties looked by VOD. Jain and Bhargava (2008) worried about the examination of questionable server mass entry retrial line with two class non-preemptive need endorsers. Juhn, and Tseng (1998) presents another telecom plot, which can bolster live recordings and decrease the holding up time to 8 minutes. McManus and Ross (1996) presented a particular transport and transmissions conspire for video - on-request (VOD) called steady - rate transmission and transport (CRTT). Maragathasundari (2015) derived the execution measures for a mass section queuing model of three periods of organization with different journey strategies. Maragathasundari, Anandapriya, Gothaiammal and Gowri (2017) described a non-markovianqueuing model in which entry was taken after a Poisson method. Maragathasundari and Karthikeyan (2016) investigated a mass queuing model with short and long escape. Maragathasundari, Srinivasan and Ranjitham (2014) examined a bunch landing queuing arrangement of stage get-away with two phases ofadministration dependent on a Bernoulli plan. Alomari and Sumari (2011) gave measurable data about the web, correspondences and cell phones and so forth. Abeywickrama and Wong (2013) featured that vital advancement of a nearby capacity inside the system empowered the 
administrations to be conveyed with improved nature of administration to the client. Kanrar (2012) displayed an effective estimation of the transfer speed necessity for the distinctive design. Gupta (2013) should attempt to respond to addresses identified with innovative and administrative difficulties looked by IPTV and the requirements identified with its effective usage in India and broke down the capability of IPTV as an apparatus of instruction in setting to the changing worldview of educating - learning strategy - and Pedagogy. Viswanathan and Imielinski (1996) gave expository and trial assessments of pyramid broadcasting dependent on its execution on an Ethernet LAN. Van Den Broeck, Pierson and Lievens (2007) orchestrated learning on existing review rehearses just as the Video-onrequests new affordances. He and Liu (2009) demonstrated that VOVO was adaptable and compelling, giving short start up latencies and great execution in VCR intuitive.

\subsection{PROCESS CARRIED OUT IN VOD PROGRAMMING}

Different applications have different performance requirements. We list here six such requirements:

\section{Batch arrival:}

In VOD, every client is appointed its own committed uncast stream. Henceforth clients appreciate fantastic adaptability in interacting with the server while viewing their videos.

\section{First Stage Service: Multicast delivery VOD service}

In a multicast conveyance VOD administration, motion pictures are made accessible just toward the start of spaces. The opening term is on the request of minutes (in our investigation we utilize the range from 30 seconds to 20 minutes). Clients making a solicitation will in this way need to pause, by and large, a large portion of a space span before the motion picture can begin. For short opening lengths (state 6 minutes) this ought not influence "onrequest" nature of the framework. At the point when the server gets a client demand it decides whether assets are accessible to support the solicitation. The server utilizes data about exceptional solicitations and the accessibility of assets to accept or reject demands. Note that the server performs clear "First Come, First Serve"scheduling. Solicitations are not appointed need, and no solicitation is denied if assets exist to service it. Clients are educated through reaction messages whether their solicitation is acknowledged or denied. 
All demands that touch base during the present space are booked or dismissed before the finish of the opening.

Emergency vacation during the time of service:

The Long starts up deferrals are considered as Emergency Vacation during the season of administration. While a long start-up postpone is bothersome, for the most part clients are eager to hold up longer under the accompanying conditions:

a. Delay guarantee: Clients might be all the more eager to pause on the off chance that they are certain that they can watch their recordings at a specific time, regardless of whether the time is potentially minutes (or even hours) after the fact. This is the rule behind postpone ensure frameworks, such as deterministic deferral (in which clients experience comparable deferral) or reservation system, in which clients save recordings to be shown at a specific later time

b. User interactions: We list two such requirements here: response time of the interactions and control granularity of the interactions. (i) Response time of the interactions (ii) Control granularity of user's interactions

c. Others: A VOD framework should offer adequate video quality. Various administrations may require distinctive video quality relying upon the class of the clients, application, and so on. Besides, the planning arrangements utilized in a server ought to be reasonable. For instance, in film on-request, a client who happens to demand a disagreeable motion picture ought not to be separated for a client mentioning an increasingly famous motion picture, if the two are charged the equivalent.

\section{Second Stage Service:}

Interactive VOD Service: In intelligent VOD services, a client viewing a motion picture will be able to control the playout of the motion picture. Client association with on-request films can be like the interactivity customers have when they lease a motion picture and watch it utilizing a video tape machine. In addition, the utilization of advanced video will empower new ideal models for intelligence. The varieties of the conventional VGR elements of delay, rewind, and quick forward. 


\section{Service Interruption:}

Delay is occurring between separate and fix process - start-up deferral:

We characterize "start-up deferral," as the holding up time from the minute when a client at first presents a video demand until the minute when the client starts to see the video. It is along these lines the absolute holding up time before the mentioned video is gushed. Clearly, start-up deferral is an irregular variable whose esteem relies upon where the client is in the line, what the client's need class is, or even the video mentioned. We recognize here start-up deferral from the reaction time of client connections. While start-up deferral is the sitting tight time for a client before the mentioned video is shown, the reaction time of client collaborations is the inertness from the season of issuing a control order to the genuine scene change in an on-going video session. In this manner, start-up postponement can be longer than the reaction time of client communications. Diverse VOD applications have distinctive least start-up postpone necessities. The necessity may rely upon to what extent a client sees the video, i.e., the solicitation's holding time.

\section{Balking during Repair:}

If the VOD server isn't reachable and for upkeep reason, the Balking of customers may occur.

\section{Reneging during delay:}

Depending on the holding up time achieved, a customer may drop its interest and leave the system (i.e., renege). The reneging behavior of the customers is a basic idea in the structure of a nearby VOD structure and the essential interest clumping plans.

\section{Compulsory Vacation after completion of the service:}

Video servers: The video servers store various motion pictures (described by their length, fame and gushing information rate) open by the clients. Every server has limited stockpiling and spilling capacities. Such assets are viewed as constantly accessible and one might say effectively paid for the accessible gushing limit might be parceled or shared among the motion pictures. In a close VOD framework, the fundamental issue is to properly allot the constrained gushing ability to the different demands by methods for bunching. 
Necessary excursion must be taken by the video server subsequent to finishing the gushing administration. To conquer the solid issue of clog in VOD programming a lining model is proposed in the present work. The lining instrument is created dependent on the likelihood circulation in various scope of correspondence. A procedure did in VOD application is totally changed into a lining issue. As clarified above in VOD application, the procedure comprises of rendering various phases of administration, a crisis excursion (upkeep work or set up time work) before the second phase of administration. After the culmination of the second phase of administration, mandatory excursion (Compulsory support work) is done. Next, because of blockage and different issues, negligible administration intrusion emerge. To fathom the intrusion, correction procedure is done. In sensible circumstances, fix procedure can't be started promptly because of different reasons. Consequently the idea of defer happens between administration interference and patch up procedure. The lining system improves the system measurements, for example, generally speaking system throughput, lessens the course delay, over hard and traffic blockage likelihood.

\subsection{QUEUING THEORY APPROACH}

The VOD lining issue is as per the following: Clients arriving in batches follows a Poisson system. Service starts and it resumes. It pursues general dissemination and it is rendered in like manner on first started things out served premise in two phases of administration. After the fulfillment of the primary phase of administration, the server takes a crisis vacation. In this course of time, maintenance work for the second phase of administration is done. What's more, server interrupts because of different reason during the season of administration. In continuation, it needs to get into repair process, however here in this circumstance, a delay process idea is been taken over between the intrusion and fix process. Additionally, to augment the up keep work of the framework a mandatory excursion is presented after the culmination of second phase of administration. The idea of Balking assumes a noticeable job in this model. Seeing the line, clients may stop the framework without joining the line. Furthermore, to that, a portion of the customers may leave the line and quit the framework because of eagerness. This procedure is known to renege and it occurs in our model during the postpone time between administration intrusion and redo process. All the characterized parameters pursue a general conveyance. 


\section{The issue is depicted by the concept of birth and death process and utilizing the steady state conditions of the model defined}

VOD application issue is understood by the strategy for beneficial variable method. For every one of the phase of administration, postpone time, fix process, crisis get-away and necessary get-away beneficial variables are used. Enduring state likelihood creating line measure, length of the line, number of clients in the framework, holding up time of the clients in the framework just as in the line are resolved. Likewise, the use factor, the time spent by the server for the administration and inactive time of the server are inferred for the characterized VOD programming issue. Numerical depiction legitimizes the model and the graphical depiction gives a sensible picture about the decisions to be taken before the startup of the organization. To deteriorate the issue in VOD programming, an undeniable endorsement is rendered close to the end, by strategies for looking at the numerical results and graphical examination of the model.

\section{MATHEMATICAL ASSUMPTIONS OF THE MODEL}

Clients arrive in groups for service with mean arrival rate $\lambda^{+}>0$.

The first order probability that a batch of i customers arrives at the system is $\lambda^{+} d_{i}$ Here $0 \leq d_{i} \leq 1$ and $\sum_{i=1}^{n} d_{i}=1$

For the first stage of service, $\theta_{n}^{(1)}(x), \eta_{1}(x)$ is the conditional probability of completion of completion of first stage of service. The probability distribution function of the first stage of service and its corresponding density function are given by $E^{*}(x)$ and $e^{*}(x)$. Hence:

$$
\eta_{1}(x)=\frac{e^{*}(x)}{1-E^{*}(x)}, \quad e^{*}(x)=\eta_{1}(s) e^{-\int_{0}^{s} \eta_{1}(x) d x}
$$

Similarly for all the other parameters, Emergency vacation $\left(M_{n}^{E}(x)\right)$, stage 2 procedure $\left(\theta_{n}^{(2)}(x)\right)$, Compulsory vacation $\left(M_{n}^{(c)}(\mathrm{x})\right)$, Delay process $\left(D_{n}(x)\right)$, Repair process we have the following functions respectively:

$$
\begin{array}{ll}
\delta_{E}(x)=\frac{a^{*}(x)}{1-S^{*}(x)}, & a^{*}(x)=\delta_{E}(s) e^{-f \delta_{E}(x) d x} \\
\delta_{2}(x)=\frac{a_{1}^{*}(x)}{1-h_{1}^{*}(x)}, & a_{1}^{*}(x)=\delta_{2}(s) e^{-\int \delta_{2}(x) d x}
\end{array}
$$




$$
\begin{gathered}
\delta_{c}(x)=\frac{w^{*}(x)}{1-W^{*}(x)}, \quad w^{*}(x)=\delta_{c}(s) e^{-\int_{0}^{s} \delta_{c}(x) d x} \\
\varphi(x)=\frac{a_{2}^{*}(x)}{1-A_{2}^{*}(x)}, \quad a_{2}^{*}(x)=\varphi(s) e^{-\int_{0}^{s} \varphi(x) d x} \\
\gamma(x)=\frac{v^{*}(x)}{1-K^{*}(x)}, \quad v^{*}(x)=\gamma(s) e^{-\int_{0}^{s} \gamma(x) d x}
\end{gathered}
$$

During delay process, the process of reneging takes place. That is the clients leave the system due to impatience with probability $\varepsilon$ after joining the Queue. Also break down occurs during the stages of service with arrival rate $\beta>0$ follows a Poisson distribution. After entering into the system, seeing the Queue, some customers may not join the Queue and they leave the system. This process of Balking occurs with probability b during the time of repair process in this Queuing system.

\section{GOVERNING EQUATIONS OF THE MODEL}

The VOD Queuing model is first defined as a set of difference differential equations:

$$
\begin{aligned}
& \frac{\partial}{\partial x} \theta_{n}^{(1)}(x)+\left(\lambda^{+}+\eta_{1}(x)+\beta\right) \theta_{n}^{(1)}(x)=\lambda^{+} \sum_{i=1}^{n} d_{i} \theta_{n-i}^{(1)}(x) \\
& \frac{\partial}{\partial x} \theta_{0}^{(1)}(x)+\left(\lambda^{+}+\eta_{1}(x)+\beta\right) \theta_{0}^{(1)}(x)=0 \\
& \frac{\partial}{\partial x} M_{n}^{E}(x)+\left(\lambda^{+}+\delta_{E}(x)\right) M_{n}^{E}(x)=\lambda^{+} \sum_{i=1}^{n} d_{i} M_{n-i}^{E}(x) \\
& \frac{\partial}{\partial x} M_{0}^{E}(x)+\left(\lambda^{+}+\delta_{E}(x)\right) M_{0}^{E}(x)=0 \\
& \frac{\partial}{\partial x} \theta_{n}^{(2)}(x)+\left(\lambda^{+}+\eta_{2}(x)+\beta\right) \theta_{n}^{(2)}(x)=\lambda^{+} \sum_{i=1}^{n} d_{i} \theta_{n-i}^{(2)}(x) \\
& \frac{\partial}{\partial x} \theta_{0}^{(2)}(x)+\left(\lambda^{+}+\eta_{2}(x)+\beta\right) \theta_{0}^{(2)}(x)=0 \\
& \frac{\partial}{\partial x} M_{n}^{(c)}(x)+\left(\lambda^{+}+\delta_{c}(x)\right) M_{n}^{(c)}(x)=\lambda^{+} \sum_{i=1}^{n} d_{i} M_{n-i}^{(c)}(x) \\
& \frac{\partial}{\partial x} M_{0}^{(c)}(x)+\left(\lambda^{+}+\delta_{c}(x)\right) M_{0}^{(c)}(x)=0 \\
& \frac{\partial}{\partial x} D_{n}(x)+\left(\lambda^{+}+\varphi(x)+\epsilon\right) D_{n}(x)=\lambda^{+} \sum_{i=1}^{n} d_{i} D_{n-i}(x)+\epsilon D_{n+1}(x) \\
& \frac{\partial}{\partial x} D_{0}(x)+\left(\lambda^{+}+\varphi(x)+\epsilon\right) D_{0}(x)=\epsilon D_{1}(x) \\
& \frac{\partial}{\partial x} k_{n}(x)+\left(\lambda^{+}+\gamma(x)\right) k_{n}(x)=\lambda^{+}(1-b) k_{n}(x)+b \lambda^{+} \sum_{i=1}^{n} d_{i} K_{n-i}(x) \\
& \frac{\partial}{\partial x} k_{0}(x)+\left(\lambda^{+}+\gamma(x)\right) k_{0}(x)=\lambda^{+}(1-b) k_{0}(x) \\
& \lambda^{+} Q=\int_{0}^{\infty} k_{0}(x) \gamma(x) d x+\int_{0}^{\infty} M_{0}^{(c)}(x) \delta_{c}(x) d x+\lambda^{+}(1-b) Q
\end{aligned}
$$




\section{Boundary conditions}

The following boundary conditions are used to solve the above equations:

$$
\begin{gathered}
\theta_{n}^{(1)}(0)=\int_{0}^{\infty} M_{n+1}^{(c)}(x) \delta_{c}(x) d x+\int_{0}^{\infty} R_{n+1}(x) \gamma(x) d x \\
\theta_{n}^{(2)}(0)=\int_{0}^{\infty} M_{n}^{E}(x) \delta_{E}(x) d x+(1-s) \int_{0}^{\infty} \theta_{n}^{(1)}(x) \eta_{1}(x) d x \\
M_{n}^{E}(0)=s \int_{0}^{\infty} \theta_{n}^{(1)}(x) \eta_{1}(x) d x \quad n \geq 0 \\
M_{n}^{(c)}(0)=\int_{0}^{\infty} \theta_{n}^{(2)}(x) \eta_{2}(x) d x \quad n \geq \\
D_{n}(0)=\beta \int_{0}^{\infty} \theta_{n}^{(2)}(x) d x=\beta \theta_{n-1}^{(2)}(x) \quad n \geq 1 \\
k_{n}(0)=\int_{0}^{\infty} D_{n}(x) \varphi(x) d x n \geq 0
\end{gathered}
$$

\section{DISTRIBUTION OF THE QUEUE LENGTH AT ANY POINT OF TIME}

To solve equations (1) to (12) for a closed form solution we follow the procedure set out below.

We multiply (1) by and sum over $\mathrm{x}$ from 1 to and add it to (2)

We get,

$$
\frac{\partial}{\partial x} \theta_{q}^{(1)}(x, z)+\left(\lambda^{+}-\lambda^{+} D(z)+\eta_{1}(x)\right) \theta_{q}^{(1)}(x, z)=0
$$

Similarly,

$$
\begin{gathered}
\frac{\partial}{\partial x} M_{q}^{(E)}(x, z)+\left(\lambda^{+}-\lambda^{+} D(z)+\delta_{E}(x)\right) M_{q}^{(E)}(x, z)=0 \\
\frac{\partial}{\partial x} \theta_{q}^{(2)}(x, z)+\left(\lambda^{+}-\lambda^{+} D(z)+\eta_{2}(x)+\beta\right) \theta_{q}^{(2)}(x, z)=0 \\
\frac{\partial}{\partial x} M_{q}^{(c)}(x, z)+\left(\lambda^{+}-\lambda^{+} D(z)+\delta_{c}(x)\right) M_{q}^{(c)}(x, z)=0 \\
\frac{\partial}{\partial x} D_{q}(x, z)+\left(\lambda^{+}-\lambda^{+} D(z)+\epsilon-\frac{\varepsilon}{z}+\varphi(x)\right) D_{q}(x, z)=0 \\
\frac{\partial}{\partial x} K_{q}(x, z)+\left(b\left(\lambda^{+}-\lambda^{+} D(z)\right)+\gamma(x)\right) K_{q}(x, z)=0
\end{gathered}
$$

Integrating (20)-(25) between limits 0 to $x$, we obtain 


$$
\begin{gathered}
\theta^{(1)}(x, z)=\theta^{(1)}(0, z) \exp \left[-\left(\lambda^{+}-\lambda^{+} D(z)\right) x-\int_{0}^{\infty} \eta_{1}(t) d t\right] \\
M^{E}(x, z)=M^{E}(0, z) \exp \left[-\left(\lambda^{+}-\lambda^{+} D(z)\right) x-\int_{0}^{\infty} \delta_{E}(t) d t\right] \\
\theta^{(2)}(x, z)=\theta^{(2)}(0, z) \exp \left[-\left(\lambda^{+}-\lambda^{+} D(z)+\beta\right) x-\int_{0}^{\infty} \eta_{2}(t) d t\right] \\
M^{(c)}(x, z)=M^{(c)}(0, z) \exp \left[-\left(\lambda^{+}-\lambda^{+} D(z)\right) x-\int_{0}^{\infty} \delta_{c}(t) d t\right] \\
D(x, z)=D(0, z) \exp \left[-\left(\lambda^{+}-\lambda^{+} D(z)+\xi-\frac{\xi}{z}\right) x-\int_{0}^{\infty} \varphi(t) d t\right] \\
K(x, z)=K(0, z) \exp \left[-b\left(\lambda^{+}-\lambda^{+} D(z)\right) x-\int_{0}^{\infty} \gamma(t) d t\right]
\end{gathered}
$$

The above set of equations (26) holds for all $x>0$

We next multiply the boundary conditions by suitable powers of $z^{n}$ and taking summation over all possible values of $n$ and using (13) we get after simplification

$$
\begin{gathered}
z \theta^{(1)}(0, z)=z \lambda^{+} b_{1}(D(z)-1)+\int_{0}^{\infty} M^{(c)}(x, z) \delta_{c}(x) d x+\int_{0}^{\infty} K(x, z) \gamma(x) d x \\
\theta^{(2)}(0, z)=\int_{0}^{\infty} M^{E}(x, z) \delta_{E}(x) d x+(1-s) \int_{0}^{\infty} \theta^{(1)}(x, z) \eta_{1}(x) d x \\
M^{E}(0, z)=s \int_{0}^{\infty} \theta^{(1)}(x, z) \eta_{1}(x) d x \\
M_{n}^{(c)}(0, z)=\int_{0}^{\infty} \theta^{(2)}(x, z) \eta_{2}(x) d x \\
D(0, z)=\beta z \int_{0}^{\infty} \theta^{(2)}(x, z) d x \\
K(0, z)=\int_{0}^{\infty} D(x, z) \varphi(x) d x
\end{gathered}
$$

Integrating (26) by parts with respect to $\mathrm{x}$, we get,

$$
\theta^{(1) *}(z)=\theta^{(1) *}(0, z)\left(\frac{1-E^{*}(g)}{g}\right), g=\lambda^{+}-\lambda^{+} D(z)
$$

Where $E^{*}(g)=\int_{0}^{\infty} e^{-\left(\lambda^{+}-\lambda^{+} D(z)\right) x} d E_{1}(x)$ is the Laplace transform of the service time of first stage.

Again multiplying (26) on both sides by $\eta_{1}(x)$ and integrating over $x$, we get

$$
\int_{0}^{\infty} \theta^{(1) *}(x, z) \eta_{1}(x) d x=\theta^{(1) *}(0, z) E^{*}(g)
$$


Similarly,

$$
\begin{gathered}
\theta^{(2)^{*}}(z)=\theta^{(2) *}(0, z)\left(\frac{1-A_{1}^{*}(h)}{h}\right), h=\lambda^{+}-\lambda^{+} D(z)+\beta \\
\theta^{(2)^{*}}(z)=\theta^{(2) *}(0, z)\left(\frac{1-A_{1}^{*}(h)}{h}\right), h=\lambda^{+}-\lambda^{+} D(z)+\beta \\
\int_{0}^{\infty} \theta^{(2) *}(x, z) \eta_{2}(x) d x=\theta^{(2) *}(0, z) A_{1}^{*}(h) \\
\int_{0}^{E *}(z)=M^{E *}(0, z)\left(\frac{1-S^{*}(g)}{g}\right) \\
M_{0}^{\infty *}(x, z) \delta_{E}(x) d x=M^{(E *)}(0, z) S^{*}(g) \\
M^{(c) *}(z)=M^{(c) *}(0, z)\left(\frac{1-W^{*}(g)}{g}\right) \\
\int_{0}^{\infty} M^{(c) *}(x, z) \delta_{c}(x) d x=M^{(E) *}(0, z) W^{*}(g) \\
D^{*}(z)=D^{*}(0, z)\left(\frac{1-A_{2}^{*}(q)}{q}\right), q=\left(\lambda^{+}-\lambda^{+} K(z)+\xi-\frac{\xi}{z}\right) \\
\int_{0}^{\infty} D^{*}(x, z) \varphi(x) d x=D^{*}(0, z) A_{2}^{*}(q) \\
K^{*}(z)=K^{*}(0, z)\left(\frac{1-K^{*}(b g)}{b g}\right)
\end{gathered}
$$

Now utilizing the above relations (38) in (32)-(37), we get

$$
\begin{gathered}
z \theta^{(1)}(0, z)=z \lambda^{+} b_{1}(D(z)-1)+s W^{*}(g) \theta^{(1) *}(0, z) E^{*}(g)+K^{*}(b g) A_{2}^{*}(q) \beta z\left(\frac{1-A_{1}^{*}(h)}{h}\right)+\left[\left(\frac{1-S^{*}(g)}{g}\right) s \theta^{(1) *}(0, z) E^{*}(g)+(1-s) \theta^{(1) *}\right. \\
\theta^{(1)}(0, z)=\frac{z \lambda^{+} b_{1}(D(Z)-1)}{z-s W^{*}(g) E^{*}(g)-K^{*}(b g) A_{2}^{*}(q) \beta z\left(\frac{1-A_{1}^{*}(h)}{h}\right)\left[\frac{1-S^{*}(g)}{g} s+(1-s)\right] E^{*}(g)}
\end{gathered}
$$

Hence we get the following from (36) using (39) 


$$
\begin{aligned}
& \theta^{(1) *}(z)=\frac{z \lambda^{*} b_{1}(D(Z)-1)}{z-s W^{*}(g) E^{*}(g)-K^{*}(b g) A_{2}^{*}(q) \beta z\left(\frac{1-\alpha^{*}(k)}{h}\right)\left[\frac{1-s^{*}(g)}{g} s+(1-s)\right] E^{*}(g)}\left[\frac{1-E^{*}(g)}{g}\right] \\
& \theta^{(2) *}(z)=\left[S^{*}(g) s+(1-s)\right] E^{*}(g) \frac{z \lambda^{*} b_{1}(D(Z)-1)\left(\frac{1-A_{j}^{*}(h)}{h}\right)}{\left.z-s W^{*}(g) E^{*}(g)-K^{*}(b)\right) A_{2}^{*}(q) \beta z\left(\frac{1-A_{h}^{*}(h)}{h}\right)\left[\frac{1-s^{*}(g)}{g} s+(1-s)\right] E^{*}(g)} \\
& M^{E^{*}}(z)=s E^{*}(g)\left(\frac{1-s^{*}(g)}{g}\right) \frac{z \lambda^{*} b_{1}(D(L)-1)}{z-s W^{*}(g) E^{*}(g)-K^{*}(b g) A_{2}^{*}(q) \beta z\left(\frac{1-1 \phi^{*}(k)}{h}\right)\left[\frac{1-s^{*}(g)}{g} s+(1-s)\right] E^{*}(g)} \\
& M^{(c) *}(z)=A_{1}^{*}(h)\left[S^{*}(g) s+(1-s)\right] E^{*}(g) \frac{z \lambda^{+} b_{1}(D(Z)-1)\left(\frac{1-W^{*}(g)}{g}\right)}{z-s W^{*}(g) E^{*}(g)-K^{*}(b g) A_{2}^{*}(q) \beta z\left(\frac{1-A_{1}^{*}(h)}{h}\right)\left[\frac{1-S^{*}(g)}{g} s+(1-s)\right] E^{*}(g)} \\
& D^{*}(z)=\beta z\left(\frac{1-A_{1}^{*}(h)}{h}\right)\left(\frac{1-A_{2}^{*}(q)}{q}\right) \frac{z \lambda^{*} b_{1}(D(Z)-1)\left[S^{*}(g) s+(1-s)\right] E^{*}(g)}{z-s W^{*}(g) E^{*}(g)-K^{*}(b g) A_{2}^{*}(q) \beta z\left(\frac{1-A^{*} * h}{h}\right)\left[\frac{1-s^{*}(g)}{g} s+(1-s)\right] E^{*}(g)} \\
& K^{*}(z)=A_{2}^{*}(q) \beta z\left(\frac{1-A_{1}^{*}(h)}{h}\right)\left[S^{*}(g) s+(1-s)\right] E^{*}(g) \frac{z \lambda^{+} b_{1}(D(Z)-1)\left(\frac{1-K^{*}(b g)}{b g}\right)}{z-s W^{*}(g) E^{*}(g)-K^{*}(b g) A_{2}^{*}(q) \beta z\left(\frac{1-A_{1}^{*}(h)}{h}\right)\left[\frac{1-S^{*}(g)}{g} s+(1-s)\right]}
\end{aligned}
$$

\section{PROBABILITY GENERATING FUNCTION OF THE QUEUE SIZE}

To find the probability generating function of the queue size,

Let, $T_{q}(z)=\theta^{(1) *}(z)+\theta^{(2) *}(z)+M^{E *}(z)+M^{(c) *}(z)+D^{*}(z)+K^{*}(z)$

$\frac{-z b(1-E(g))+S(g) s+(1-s)] E^{*}(g) z \lambda^{*} b_{1}(D(Z)-1)\left(\frac{1-A^{*}(h)}{h}\right)-z b E^{*}(g)\left[1-S^{*}(g)\right] s-z b A_{1}^{*}(h)\left[S^{*}(g) s+(1-s)\right] E^{*}(g)\left(1-W^{*}(g)\right)+\beta z z^{*}(g) z b \lambda^{+}(D(Z)-1)\left(\frac{1-A^{*}(h)}{h}\right)\left(\frac{1-A_{2}^{*}(q)}{q}\right)\left[S^{*}(g) s+(1-s)\right]-A_{2}^{*}}{\left.z-s W^{*}(g) E^{*}(g)-K^{*}(b g) A_{2}^{*}(q)\right] z\left(\frac{1-A^{*}(k)}{h}\right)\left[\frac{\left[-S^{*}(g)\right.}{g} s+(1-s)\right] E^{*}(g)}$

\section{IDLE TIME AND UTILIZATION FACTOR}

The normalization condition $T_{q}(1)+Q=1$ is used in order to determine $Q$. Because of the indetermine of $T_{q}(1)$, L'Hopital's rule is applied in (40) to achieve

$$
T_{q}(z)=\frac{N^{\prime}(1)}{D^{\prime}(1)}
$$

Now adding $Q$ to $T_{q}(z)$ given in equation (41) and equating to 1 and simplifying we obtain

$$
Q=\frac{D^{\prime}(1)}{D^{\prime}(1)+N^{\prime}(1)}
$$

Mean length of the Queue and to find $L_{q}$, the steady state average queue length, where

$$
L_{q}=\frac{d}{d z} T_{q}(z) \mid z=1
$$

We note that this formula is of $\frac{0}{0}$ form. 
Hence we write $T_{q}(z)$ as $T_{q}(z)=\frac{N(z)}{D(z)}$ where $\mathrm{N}(\mathrm{z})$ and $\mathrm{D}(\mathrm{z})$ are the numerator and denominator of equation (40)

Then using L'Hopital's rule, we obtain

$$
L_{q}=\lim _{z \rightarrow 1} \frac{D^{\prime}(z) N^{\prime \prime}(z)-N^{\prime}(z) D^{\prime \prime}(z)}{2\left(D^{\prime}(z)\right)^{2}}
$$

Finding the required derivatives at $\mathrm{z}=1$, we have

$$
\begin{gathered}
N^{\prime}(1)=b_{1}\left\{\left(-b_{1} E(R) \lambda^{+}+1\right)\left(\frac{1-A_{i}^{*}(\beta)}{\beta}\right)+E(W) \lambda^{+}\right\}-b_{1}\left[\lambda^{+}+\lambda^{+} s E(s)\right] \\
D^{\prime}(1)=1+s \lambda^{+}[E(L)+E(W)]-\left(1-A_{1}^{*}(\beta)\right)(1-s)\left\{\left[\left(b E(R) \lambda^{+}+R^{*}(b) E(L)\right)\left(\lambda^{+}\right)\right]+\left[-E\left(A_{2}\right)\left(-\lambda^{+}+\xi\right)+1\right] R^{*}(b)\right\}-R^{*}(b) \\
N^{\prime \prime}(1)=\left(2 b_{1} s \lambda^{+} E(s)+2 b_{1} E(L) \lambda^{+}+2 b_{1}\right)\left[\left(-\lambda^{+} b_{1} E(R)+\lambda^{+}\right)\left(\frac{1-A_{i}^{*} \beta}{\beta}\right)+E(W) \lambda^{+}\right]+\left[b_{1} \frac{\beta}{b_{1}} E(R)+\left(-\lambda^{+}+\xi\right)\left(-\lambda^{+}\right) E(R)+\lambda^{+} \beta\right. \\
D^{\prime \prime}(1)=-s\left(\lambda^{+}\right)^{2}\left[2 E(W) E(L)+E\left(L^{2}\right)+E\left(W^{2}\right)\right]-b_{1}\left(\lambda^{+}\right)^{2} E\left(R^{2}\right)\left(1-A_{1}^{*}(\beta)\right)(1-s)+E\left(A_{2}\right)\left(\lambda^{+}\right)^{2} E(R)\left(1-A_{1}^{*}(\beta)\right)(1-s)+E(R) b
\end{gathered}
$$

Substituting (44) in (43) we obtain $L_{q}$ in closed form.

Further, the man waiting time of the customers in the queue as well as in the system and number of customers waiting in the system can be found using Little's law

$$
W_{q}=\frac{L_{q}}{\lambda^{+}}, \mathrm{W}=\frac{L}{\lambda^{+}}, \mathrm{L}=L_{q}+\rho
$$

\section{NUMERICAL JUSTIFICATION OF THE MODEL}

Assume that service time follows exponential distribution in particular and based on this condition, the numerical justification is elaborated below. The values are collected accordingly: $\lambda^{+}=3, \beta=4, b_{1}=1, \varphi=3, \gamma=2.5, \mathrm{~s}=0.5, \eta_{1}=4, \eta_{2}=5, \delta_{E}=3.5, \delta_{c}=2$

$$
\begin{aligned}
& E(L)=\frac{1}{\eta_{1}}, E\left(L^{2}\right)=\frac{2}{\eta_{1}}, E(s)=\frac{1}{\delta_{E}}, E\left(s^{2}\right)=\frac{2}{\delta_{E^{2}}}, \quad E(W)=\frac{1}{\delta_{c}}, \quad E\left(W^{2}\right)=\frac{2}{\delta_{c}^{2}}, \quad A_{1}^{\prime}(\beta)=\frac{-\eta_{2}}{\left(\eta_{2}+\beta\right)^{2}}, \\
& E\left(A_{2}\right)=\frac{1}{\varphi}, E\left(A_{2}^{2}\right)=\frac{2}{\varphi^{2}}, E(R)=\frac{1}{\gamma}, E\left(R^{2}\right)=\frac{2}{\gamma^{2}}, R^{*}(b)=1, A_{1}^{*}(\beta)=\frac{\eta_{2}}{\eta_{2}+\beta}
\end{aligned}
$$

Table 1. Effect Of ChangeOf Reneging ( $\xi=1,1.5,2,2.5,3)$.

\begin{tabular}{|c|c|c|c|c|c|}
\hline $\boldsymbol{Q}$ & $\boldsymbol{\rho}$ & $\boldsymbol{L}$ & $\boldsymbol{L}$ & $\boldsymbol{W}_{\boldsymbol{q}}$ & $\boldsymbol{W}$ \\
\hline 0.4116 & 0.5884 & 7.9348 & 8.5232 & 2.8411 & 2.6449 \\
\hline 0.4197 & 0.5803 & 7.4296 & 8.0099 & 2.67 & 2.4765 \\
\hline 0.4275 & 0.5725 & 6.9683 & 7.5408 & 2.5136 & 2.3228 \\
\hline 0.4352 & 0.5648 & 6.5741 & 7.1389 & 2.3796 & 2.1914 \\
\hline 0.4426 & 0.5574 & 6.1635 & 6.7209 & 2.2403 & 2.0545 \\
\hline
\end{tabular}




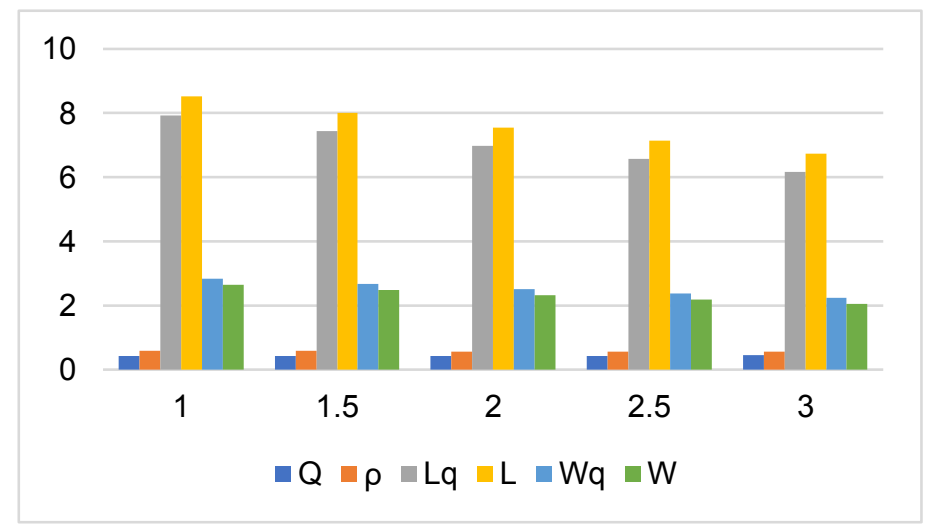

Graphic 1. Effect of change of Reneging.

Table 2. Effect Of Change Of Breakdown ( $\beta=1.5,2,2.5,3,3.5)$.

\begin{tabular}{|c|c|c|c|c|c|}
\hline$Q$ & $\rho$ & $L_{q}$ & $L$ & $W_{Q}$ & $W$ \\
\hline 0.4705 & 0.5295 & 2.1088 & 2.6383 & 0.7029 & 0.8794 \\
\hline 0.4537 & 0.5463 & 2.7691 & 3.3154 & 0.9230 & 1.1051 \\
\hline 0.4484 & 0.5516 & 3.1270 & 3.6786 & 1.0423 & 1.2262 \\
\hline 0.4209 & 0.5791 & 4.0008 & 4.5799 & 1.3336 & 1.5266 \\
\hline 0.4141 & 0.5859 & 4.4935 & 5.0794 & 1.4978 & 1.6931 \\
\hline
\end{tabular}

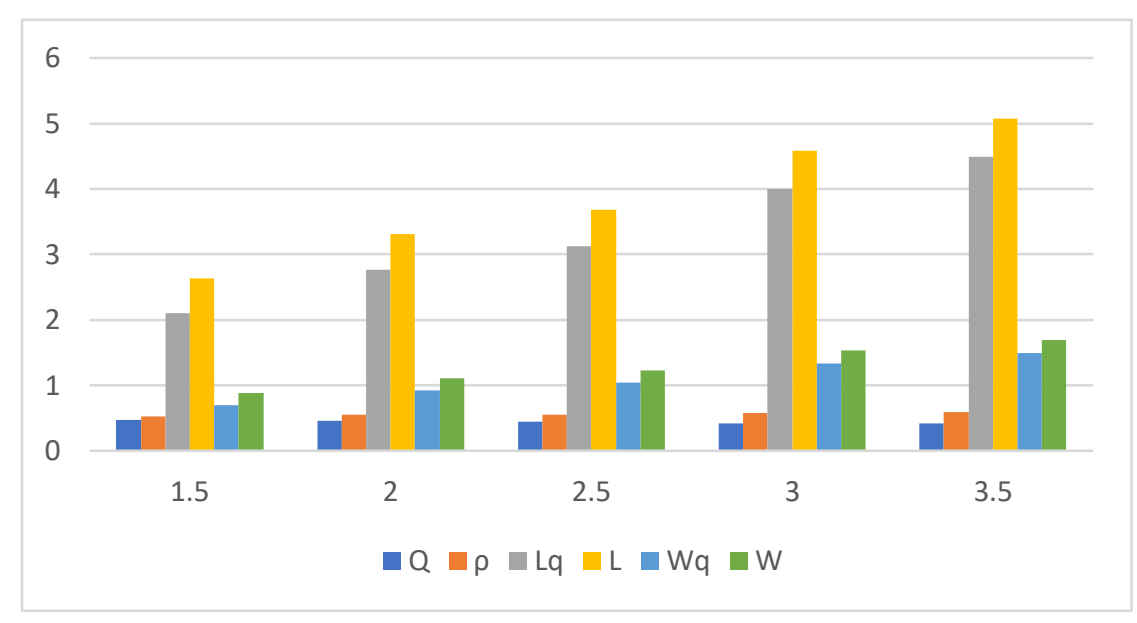

Graphic 2. Effect of change of Breakdown.

From the Table 1, the fact is clear that, as the service goes on in the system, the process of reneging factor occurring during delay process increases. This creates an effect in all the Queue execution measures. It leads to an increase in the idle time and hence the utilization 
factor decreases. Length of the Queue, number of customers in the system and the waiting time gets decreased.

Next, Table 2 shows the performance measures of the system when the break down factor increases. It leads to an increase in all the performance measures of the system as expected. The Idle time dropped off and it leads to an enlarge the utilization factor.

\section{CONCLUSION}

This VOD application process clearly defines the Queuing model consisting of the parameters Stages of service, multi vacation policy, Delay process, service interruption, revamp process, Balking and Reneging. VOD service is well analyzed by means of Queuing approach and the problem is solved by supplementary variable method. Queue performance measures are derived and the model is well justified by the way of numerical illustration. All the results are as expected. 


\section{REFERENCES}

Abeywickrama, S., \& Wong, E. (2013). Delivery of video-on-demand services using local storages within passive optical networks. Optics Express, 21(2), 2083-2096. https://doi. org/10.1364/OE.21.002083

Alomari, S. A., \& Sumari, P. (2011). Statistical information of the Increased demand for watch the VOD with theincreased sophistication in the mobile devices,communications and internet penetration in Asia. The International fournal of Multimedia and its Applications, 3(4), 183-199. https://arxiv.org/ftp/arxiv/papers/1112/1112.2042.pdf

Ghimire, S., Ghimire, R., Thapa, G. B., \& Fernandes, S. (2017). Multi-server batch service queuing model with variable service rates. International fournal of Applied Mathematics and Statistical Science, 6(4), 43-54. https://www.researchgate.net/ publication/321135050_MULTI-SERVER_BATCH_SERVICE_QUEUEING_ MODEL_WITH_VARIABLE_SERVICE_RATES

Gupta, S. (2013). IPTV: A new dimension in online video streaming - A study of the Indian scenario. Global Media Fournal, 4(1), 1-17. https://www.caluniv.ac.in/globalmdia-journal/CMENT_JUNE_2013/COM-1-Sourav.pdf

He, Y., \& Liu, Y. (2009). VOVO: VGR-oriented Video-on-Demand in Large scale peerto-peer networks. IEEE Transactions on Parallel and Distributed systems, 20(4), 528-539. https://doi.org/10.1109/TPDS.2008.102

Jain, M., \& Bhargava, G. (2008). Bulk arrival retrial queue with unreliable server and priority subscribers. International Fournal of Operational Research, 5(4), 242-259. https:// www.researchgate.net/publication/266828621_Bulk_Arrival_Retrial_Queue_ with_Unreliable_Server_and_Priority_Subscribers

Juhn, L. S., \& Tseng, L. M. (1998). Fast Data broadcasting and receiving scheme for popular video service. IEEE Transactions on Broadcasting, 44(1), 100-105. https://doi. org/10.1109/11.713059 
Kanrar, S. (2012). Analysis and implementation of the large scale video-on-demand system. International Journal of Applied Information Systems, 2(12), 1-9. https://arxiv.org/ $\mathrm{ftp} /$ arxiv/papers/1202/1202.5094.pdf

Maragathasundari, S. (2015). A Bulk arrival queuing model of three stages of service with different vacation policies service interruption and delay time. American International Journal of Research in Science and Technology, 11(1), 52-56. https://www.researchgate. net/publication/295605742_A_bulk_arrival_queueing_model_of_three_stages_of_ service_with_different_vacation_policies_service_interruption_and_delay_time

Maragathasundari, S., \& Karthikeyan, K. (2016). A Bulk queuing model of Optional second phase service with short and long vacations. International fournal of Scientific Research in Science and Technology, 2(5), 196-201. https://www.academia. edu/29971131/A_Bulk_Queuing_Model_of_Optional_Second_Phase_Service_ with_Short_and_Long_Vacations

Maragathasundari, S., Anandapriya, B., Gothaiammal, S. B., \& Gowri, V. (2017). M/G/1 Queue with restricted availability during service interruption and compulsory vacation of deterministic time. International Fournal of Mathematics Trends and Technology, 52(1), 5-9. http://www.ijmttjournal.org/2017/Volume-52/number-1/IJMTTV52P502.pdf

Maragathasundari, S., Srinivasan, S., \& Ranjitham, A. (2014). Analysis of a batch arrival queue with two stages of service and phase vacation. Missouri fournal of Mathematical Sciences, 26(2), 189-205. https://doi.org/10.35834/mjms/1418931959

McManus, J. M., \& Ross, K. W. (1996). Video-on-demand over ATM: Constant-rate transmission and transport. IEEE Journal on selected areas in Communication, 14(6), 10871098. https://doi.org/10.1109/49.508280

Singh Walia, A. (2016). Live multimedia streaming and video on demand issues and challenges. International Journal of Research in Engineering and Technology, 5(2), 91-96. https://www.academia.edu/25603526/LIVE_MULTIMEDIA_STREAMING_ AND_VIDEO_ON_DEMAND_ISSUES_AND_CHALLENGES 
Van Den Broeck, W., Pierson, J., \& Lievens, B. (2007). Video-On-Demand: Towards New Viewing Practices? Observatorio Fournal, 3, 23-44. http://citeseerx.ist.psu.edu/ viewdoc/download?doi=10.1.1.1011.3016\&rep=rep 1\&type=pdf

Viswanathan, S., \& Imielinski, T. (1996). Metropolitan area video-on-demand service using pyramid broadcasting. Multimedia Systems, 4(4), 197-208. https://doi. org/10.1007/s005300050023 
